ORIGINAL ARTICLE

\title{
DT5aP-Hib-IPV and MCC vaccines: preterm infants' response to accelerated immunisation
}

\author{
M H Slack, S Cade, D Schapira, R J Thwaites, A Crowley-Luke, J Southern, R Borrow, E Miller
}

Arch Dis Child 2005;90:338-341. doi: 10.1136/adc.2004.052720

See end of article for authors' affiliations

Correspondence to:

Dr M Slack, Department of

Paediatrics, St Mary's

Hospital, Portsmouth PO3

6AD, UK; marts@doctors.

org.uk

Accepted 28 August 2004

preterm infants to combined diphtheria/tetanus/ 5 component Aims: To describe the immune response of preterm infants to combined diphtheria/tetanus $/ 5$ component
acellular pertussis-Haemophilus influenzae type $b$ inactivated polio vaccine (DT5aP-Hib-IPV) and acellular pertussis-Haemophilus influenzae type $b$ inactivated polio vaccine (DTSaP-Hib-IPV) and
meningococcal serogroup $C$ conjugate vaccine (MCC) under accelerated schedule. To compare results with term infants immunised with DT5aP-Hib-IPV and with historical data from preterm infants immunised with a DT3 component aP-Hib vaccine.

Methods: Prospective observational study in preterm infants born at $<32$ weeks gestation with comparison to contemporary cohort of term infants. DT5aP-Hib-IPV and MCC vaccines were given at 2,3 , and 4 months.

Results: Fifty preterm infants (mean gestational age 28.5 weeks) completed the study. After three doses of vaccines Hib polysaccharide lgG geometric mean concentration (GMC) was $1.21 \mu \mathrm{g} / \mathrm{ml}$ with $80 \%$ $\geqslant 0.15 \mu \mathrm{g} / \mathrm{ml}$; MCC serum bactericidal assay geometric mean titre (GMT) was 1245 with $100 \% \geqslant 8$. All infants achieved protective titres to diphtheria, tetanus, and the three poliovirus types with $\geqslant 80 \%$ achieving protective rises in $\lg G$ against the five pertussis antigens.

Conclusion: Preterm infants immunised with DT5aP-Hib-IPV and MCC vaccines show lgG responses to Hib and MCC greater than seen historically in both term and preterm infants with a DT3aP-Hib vaccine, and for pertussis antigens and poliovirus type 1 responses similar to that seen in term infants immunised with DT5aP-Hib-IPV. Responses to poliovirus types 2 and 3 are reduced, but all infants achieved protective titres.

$\mathrm{P}$

reterm infants mount an immune response to the antigens contained in combined diphtheria/tetanus/ whole cell pertussis (DTwP) vaccines equivalent to that seen in term infants, under differing schedules. ${ }^{1-4}$ In addition, we have shown acceptable responses to the protein antigens of a DT-3 component acellular pertussis (aP) vaccine given under an accelerated schedule. ${ }^{5}$ Immune responses to inactivated polio vaccine (IPV) and oral polio vaccine (OPV) are similar to those seen in term infants, ${ }^{67}$ and preterm infants are able to mount a protective neutralising antibody response to IPV given in the first 24 hours of life. ${ }^{8}$ The proportion of preterm infants achieving protective titres against poliovirus type 3, however, may be reduced when a schedule using both IPV and OPV is used. ${ }^{9}$

There is, however, concern regarding the ability of preterm infants to respond adequately to polysaccharide conjugate vaccines given in early life. The response to Haemophilus influenzae type b polyribosylphosphate (PRP) conjugated to tetanus-toxoid (PRP-T) and combined with an aP vaccine is reduced in term and preterm infants compared to that seen with combined wP-Hib vaccines, the reduction being greater in preterm infants. ${ }^{10}$ However, both the immunogenicity and efficacy of a heptavalent pneumococcal conjugate vaccine ${ }^{11}$ and the immunogenicity of a meningococcal serogroup $\mathrm{C}$ polysaccharide-CRM ${ }_{197}$ conjugate vaccine have been shown to be comparable to that seen in term infants. ${ }^{10}$

The reduced response to PRP-T when combined with DTaP has been described previously in term infants immunised with aluminium hydroxide adjuvanted DTaP vaccines. ${ }^{12}{ }^{13}$ One DTaP vaccine containing aluminium phosphate as an adjuvant does not appear to show the same interference with the anti-PRP response, ${ }^{14}$ and it has been postulated that the attenuation in PRP response seen in infants immunised with aP-Hib vaccines follows deconjugation of Hib PRP from the carrier protein, catalysed by the aluminium hydroxide adjuvant.
We have investigated the response of preterm infants to a combined DT5aP-Hib-inactivated polio vaccine (IPV) containing aluminium phosphate adjuvant given at the same time as a meningococcal serogroup $\mathrm{C}$ vaccine under the United Kingdom's accelerated schedule.

\section{METHODS}

Infants born at less than 32 weeks gestation were recruited from three UK neonatal units: St Mary's Hospital, Portsmouth; Royal Hampshire County Hospital, Winchester; and Princess Anne Hospital, Southampton. The study had local research ethics committee approval in all three centres and took place between May 2002 and March 2003. Informed consent was obtained from the parents of the infants involved.

As part of a larger randomised study, term infants were recruited from immunisation clinics in Hertfordshire and Gloucestershire, UK. This study was approved by the London Multi-centre Research Ethics Committee and by the local ethics committees for the sites involved.

All infants were immunised according to the UK accelerated schedule $(2,3,4$ months of age). The study nurses immunised preterm infants either on the neonatal unit or at home, and term infants in immunisation clinics. DT5aP-HibIPV (Pediacel, Aventis Pasteur MSD, $0.5 \mathrm{ml}$ dose containing $20 \mu \mathrm{g}$ pertussis toxin (PT), $20 \mu \mathrm{g}$ filamentous haemagluttinin (FHA), $5 \mu \mathrm{g}$ agluttinins 2 and 3 (Fim2\&3), $3 \mu \mathrm{g}$ Pertactin $(\mathrm{PRN}), \geqslant 30$ IU purified diphtheria toxoid, $\geqslant 40$ IU purified tetanus toxoid, 40D antigen units of poliovirus type I, 8D Abbreviations: aP, acellular pertussis; DT5aP-Hib-IPV, diphtheria/
tetanus/5 component acellular pertussis-Haemophilus influenzae type b
inactivated polio vaccine; DTwP, diphtheria/tetanus/whole cell
pertussis; GMC, geometric mean concentration; GMT, geometric mean
titre; IPV, inactivated polio vaccine; MCC, meningococcal serogroup C
conjugate vaccine; OPV, oral polio vaccine; PRP, polyribosylphosphate 
antigen units of poliovirus type II, 32D antigen units of poliovirus type III, and equivalent of $10 \mu \mathrm{g}$ of Hib polysaccharide conjugated to tetanus toxoid) was given into the right thigh. MCC (Meningitec, Wyeth, $0.5 \mathrm{ml}$ dose containing $10 \mu \mathrm{g}$ meningococcal serogroup $\mathrm{C}$ oligosaccharide conjugated to approximately $15 \mu \mathrm{g} \mathrm{CRM}_{197}$ protein) was given into the left thigh of the preterm infants. Menjugate (Chiron, $0.5 \mathrm{ml}$ dose containing $10 \mu \mathrm{g}$ meningococcal serogroup $\mathrm{C}$ oligosaccharide conjugated to approximately $15 \mu \mathrm{g}^{\mathrm{CRM}} 197$ protein) was given into the left thigh of the term infants. Where postthird dose PRP IgG concentration was $<1.0 \mu \mathrm{g} / \mathrm{ml}$ a fourth additional dose of Hib conjugate vaccine (Hiberix, GlaxoSmithKline, containing $10 \mu \mathrm{g}$ of Hib polysaccharide conjugated to tetanus toxoid) was given.

\section{Assays}

Blood was taken by needle venepuncture into Microtainer Serum Separator tubes just prior to the first and 4-6 weeks after the third vaccine was given. Antibody concentrations to PRP, diphtheria, and tetanus were assayed at HPA Porton Down by enzyme linked immunosorbent assay (ELISA). Serum bactericidal assay (SBA) to MCC was determined by the HPA Meningococcal Reference Unit, Manchester using rabbit complement according to established techniques. ${ }^{15}$ Antibody concentrations to the pertussis antigens (FHA, Fim2\&3, PRN, PT) were determined by ELISA and neutralising antibody titres to poliovirus types 1, 2, and 3 by neutralisation assay at the laboratories of AventisPasteur, USA. Where compared, assays for term and preterm infants were performed in the same laboratories.

In infants where there was insufficient serum available for all vaccine antigen assays to be performed, the order of priority (decreasing) was as follows: pertussis antigens, PRP, MCC, tetanus, poliovirus type 1, diphtheria, and poliovirus types 2 and 3.

\section{Statistical analysis}

Antibody concentrations and titres were $\log _{10}$ transformed to achieve normality. PRP geometric mean concentration (GMC) and diphtheria, tetanus, FHA, Fim2\&3, PRN and PT IgG, MCC SBA, and poliovirus types 1, 2, and 3 neutralising antibody geometric mean titres (GMT) were calculated with 95\% confidence intervals (95\% CI). For computational ease infants with undetectable PRP responses were given a value of $0.08 \mu \mathrm{g} / \mathrm{ml}$ and a titre of 2 for undetectable MCC SBA responses. Single variable linear regression analysis was performed to evaluate the influence of gestational age and weight at birth, pre-immunisation IgG levels, age at third immunisation, and number of doses of antenatal steroids on the IgG response to each of the vaccine antigens. Variables with a p value $<0.2$ in single variable analysis were examined further using multivariable analysis.

\section{RESULTS}

Sixty term infants and 55 preterm infants were recruited to the study. Of the preterm infants, 50 completed their primary immunisations. Of the five who did not, two died following first immunisation, one from chronic lung disease and one from non-accidental injury. Two further infants were withdrawn from the study by their parents: one following an Epstein-Barr virus associated encephalitis and one who became unsettled in the week following the second immunisation. One infant was excluded following immunisation outside the study.

Mean gestational age at birth of the preterm infants who completed the study was 28.5 weeks (range 23.9-31.9 weeks) and mean birth weight $1114 \mathrm{~g}$ (range 520-2020 g). Mean age and weight at first immunisation were 63 days (range 5489 days) and $2367 \mathrm{~g}$ (range 1230-4000 g) respectively.

For the preterm infants, PRP IgG GMC prior to first immunisation was $0.14 \mu \mathrm{g} / \mathrm{ml}(95 \%$ CI 0.11 to $0.17 \mu \mathrm{g} / \mathrm{ml})$. This rose to $1.21 \mu \mathrm{g} / \mathrm{ml}$ (95\% CI 0.73 to $2.03 \mu \mathrm{g} / \mathrm{ml})$ following third immunisation $(\mathrm{p}<0.0001)$. These results, and the proportions achieving protective titres, are shown in table 1. Of note, $20 \%$ of preterm infants had undetectable PRP IgG after the three primary immunisations and $40 \%$ had a concentration $<1.0 \mu \mathrm{g} / \mathrm{ml}$. Sixteen infants with a PRP IgG $<1.0 \mu \mathrm{g} / \mathrm{ml}$ after the three primary immunisations received an additional fourth dose of Hib at a mean age of 6.6 months (range 5.6-8.0 months). PRP IgG GMC of these infants was $0.20 \mu \mathrm{g} / \mathrm{ml}$ (95\% CI 0.11 to $0.37 \mu \mathrm{g} / \mathrm{ml})$ post-third dose, rising to $1.09 \mu \mathrm{g} / \mathrm{ml}(95 \%$ CI 0.44 to $2.68 \mu \mathrm{g} / \mathrm{ml})$ one month after a fourth dose $(p=0.002)$. After the 4 th, additional, dose of Hib, six of the 16 infants still had a PRP IgG concentration $<1.0$, with three infants $<0.15 \mu \mathrm{g} / \mathrm{ml}$.

Four infants received postnatal steroids for chronic lung disease. In two of these there was insufficient blood to determine PRP IgG post-third dose. The remaining two infants had PRP IgGs post-third dose of 3.67 and $1.67 \mu \mathrm{g} / \mathrm{ml}$.

In the preterm infants MCC SBA GMT prior to first immunisation was 2.51 (95\% CI 2.05 to 3.07). This rose to 1245.10 (95\% CI 745.62 to 2079.20 ) following third immunisation $(\mathrm{p}<0.0001)$, with $100 \%$ of infants achieving a SBA titre $\geqslant 1: 8$. No significant association was found between MCC SBA response and patient variables.

The GMC/Ts and proportions achieving protective titres for Hib and MCC with Pediacel in this study and those achieved after three doses of a three component DTaP-Hib vaccine (Infanrix-Hib, GlaxoSmithKline) in a previous study ${ }^{10}$ are shown in table 1.

GMTs for the pertussis antigens FHA, Fim2\&3, PRN, and PT, and for poliovirus types 1, 2, and 3 are shown in table 2 . GMTs for these antigens achieved in term infants after three doses of Pediacel with MCC vaccine given concurrently at 2, 3 , and 4 months are given for comparison.

\begin{tabular}{|c|c|c|}
\hline & $\begin{array}{l}\text { Pediacel } \\
\text { PRP: } n=45 ; \text { MCC: } n=39\end{array}$ & $\begin{array}{l}\text { Infanrix-Hib } \\
\text { PRP: } n=103 ; \text { MCC } n=105\end{array}$ \\
\hline $\begin{array}{l}\text { PRP IgG GMC } \mu \mathrm{g} / \mathrm{ml}(95 \% \mathrm{Cl}) \\
\mathrm{lgG} \geqslant 0.15 \mu \mathrm{g} / \mathrm{ml} \\
\mathrm{lgG}>1.0 \mu \mathrm{g} / \mathrm{ml} \\
M C C \text { SBA GMT }(95 \% \mathrm{Cl}) \\
\mathrm{SBA} \geqslant 8 \\
\mathrm{SBA} \geqslant 128\end{array}$ & $\begin{array}{l}1.21(0.73 \text { to } 2.03) \\
36(80 \%) \\
27(60 \%) \\
1245(746 \text { to } 2079) \\
39(100 \%) \\
36(92 \%)\end{array}$ & $\begin{array}{l}0.27(0.21 \text { to } 0.35) \\
57(55 \%) \\
22(21 \%) \\
398(298 \text { to } 532) \\
104(99 \%) \\
93(86 \%)\end{array}$ \\
\hline
\end{tabular}


Table 2 GMTs for FHA, Fim 2\&3, PT, PRN, diphtheria, tetanus, and poliovirus types 1, 2, and 3 with $95 \%$ Cls and proportions achieving protective cut off levels after three doses of Pediacel for both preterm and term infants

\begin{tabular}{|c|c|c|c|c|c|c|c|}
\hline & $\begin{array}{l}\mathrm{FHA} \\
\mathrm{EU} / \mathrm{ml} \\
\% \geqslant 4 \times \text { rise }\end{array}$ & $\begin{array}{l}\mathrm{Fim} 2 \& 3 \\
\mathrm{EU} / \mathrm{ml} \\
\% \geqslant 4 \times \text { rise }\end{array}$ & $\begin{array}{l}\mathrm{PT} \\
\mathrm{EU} / \mathrm{ml} \\
\% \geqslant 4 \times \text { rise }\end{array}$ & $\begin{array}{l}\mathrm{PRN} \\
\mathrm{EU} / \mathrm{ml} \\
\% \geqslant 4 \times \text { rise }\end{array}$ & $\begin{array}{l}\text { Poliovirus type } 1 \\
\text { Titre } \\
\% \geqslant 1: 8\end{array}$ & $\begin{array}{l}\text { Poliovirus type } 2 \\
\text { Titre } \\
\% \geqslant 1: 8\end{array}$ & $\begin{array}{l}\text { Poliovirus type } 3 \\
\text { Titre } \\
\% \geqslant 1: 8\end{array}$ \\
\hline \multicolumn{8}{|l|}{ Preterm } \\
\hline $\begin{array}{l}\text { GMT } \\
\text { (95\% Cl) }\end{array}$ & $\begin{array}{l}65.91 \\
(55.31 \text { to } 78.53)\end{array}$ & $\begin{array}{l}242.13 \\
\text { (178.27 to } 328.87 \text { ) }\end{array}$ & $\begin{array}{l}76.82 \\
(62.35 \text { to } 94.64)\end{array}$ & $\begin{array}{l}38.83 \\
(26.43 \text { to } 57.04 \text { ) }\end{array}$ & $\begin{array}{l}258.79 \\
\text { (185.39 to } 361.25 \text { ) }\end{array}$ & $\begin{array}{l}285.80 \\
(162.93 \text { to } 501.34)\end{array}$ & $\begin{array}{l}287.34 \\
(171.32 \text { to } 481.94)\end{array}$ \\
\hline n & & 50 & 50 & 50 & 32 & 22 & 12 \\
\hline$\%$ & $90 \%$ & $96 \%$ & $92 \%$ & $80 \%$ & $100 \%$ & $100 \%$ & $100 \%$ \\
\hline \multicolumn{8}{|l|}{ Term } \\
\hline GMT & 48.1 & 309.0 & 86.4 & 34.1 & 338.8 & 867.6 & 1087 \\
\hline (95\% Cl) & (39.4 to 50.3 ) & (248.5 to 285.5 ) & (68.5 to 109.0 ) & (26.0 to 44.7 ) & (216.4 to 530.5 ) & (508.0 to 1481.9 ) & (700.4 to 1688.8 ) \\
\hline & $67 \%$ & $88 \%$ & $82 \%$ & $73 \%$ & $100 \%$ & $100 \%$ & $97.8 \%$ \\
\hline
\end{tabular}

Diphtheria IgG GMC after third primary immunisation in preterm infants was $1.39 \mathrm{IU} / \mathrm{ml}(\mathrm{n}=27,95 \%$ CI 0.98 to 1.98 , $100 \% \geqslant 0.1 \mathrm{IU} / \mathrm{ml})$ and IgG GMC to tetanus was $1.47 \mathrm{IU} / \mathrm{ml}$ ( $\mathrm{n}=35,95 \%$ CI 1.10 to $1.96,100 \% \geqslant 0.01 \mathrm{IU} / \mathrm{ml}$ ).

In single variable linear regression analysis each patient variable gave a $\mathrm{p}$ value $<0.2$ for at least one IgG response and so all were included in the multivariable model. No significant association was found between variables and IgG responses to PRP, MCC, FHA, Fim 2\&3, and poliovirus types 1, 2, and 3. The IgG response to diphtheria was correlated negatively with gestational age at birth $(\mathrm{p}<0.001)$ and increasing number of doses of antenatal steroids given to the infants' mothers $(p=0.01)$ and positively with birth weight $(p=0.001)$. IgG response to tetanus was correlated negatively to both gestational age at birth and pre-immunisation levels of anti-tetanus toxoid IgG $(p=0.002$ and $p=0.003$ respectively). IgG response to PRN was correlated negatively to pre-immunisation anti-PRN IgG levels and that to PT to birth weight $(\mathrm{p}<0.01$ and $\mathrm{p}=0.02$ respectively).

\section{DISCUSSION}

The availability of a combined DT5aP-Hib-IPV vaccine that elicits protective responses to its various antigens presents an attractive choice for use in the preterm infant population. Acellular pertussis vaccines have a reduced side effect profile compared to whole cell pertussis vaccines and those containing five pertussis components have been shown to have an efficacy against pertussis infection equivalent to that seen with a UK whole cell pertussis vaccine. ${ }^{16}$ Because of the problems of giving oral polio vaccine on the neonatal unit, polio immunisation is frequently delayed and falls out of synchrony with the other vaccines in the primary schedule with the risk of doses being missed. The provision of an IPV vaccine combined with DTaP-Hib thus has obvious advantages.

Before recommending the use of a combined DTaP-Hib vaccine a key issue must be addressed, and that is whether such a combined vaccine stimulates a sufficient response to Hib. The use of a DT3aP-Hib vaccine (Infanrix-Hib) resulted in very low levels of PRP IgG in preterm infants. ${ }^{10}$ In addition, the use of this vaccine has been associated with an increased relative risk of invasive Hib disease, despite full immunisation history, compared to combined whole cell pertussis-Hib preparations. ${ }^{17}$ In this study we have shown that preterm infants immunised with a DT5aP-Hib-IPV vaccine (Pediacel) achieve a Hib IgG GMC greater than that seen previously in both term and preterm infants with Infanrix-Hib. ${ }^{10}$ Whether this observed difference is due to the use of an aluminium phosphate adjuvant in Pediacel compared to aluminium hydroxide in Infanrix-Hib, or to the addition of the fimbrial proteins in Pediacel is unclear. However, while the response to Hib with Pediacel is greater than that seen with InfanrixHib, a significant minority of infants had IgG concentrations below protective levels after three doses of Pediacel $(20 \%$ $<0.15$ and $40 \%<1.0 \mu \mathrm{g} / \mathrm{ml}$ ). We would recommend, therefore, that when Pediacel is used in an accelerated schedule provision should be made for the administration of a fourth additional dose of Hib conjugate in this group of infants.

We have again shown an acceptable response to an MCC vaccine conjugated to $\mathrm{CRM}_{197}$ (Meningitec) in preterm infants immunised under an accelerated schedule. Of note, the SBA response to MCC seen in this study is increased compared to that seen when the same vaccine was given with Infanrix-Hib. ${ }^{10}$ Whether this represents a MCC batch associated difference or an enhancement effect when given with Pediacel is unclear.

The IgG responses to the pertussis components are similar to those achieved in term infants with Pediacel while those to poliovirus types 2 and 3 are reduced. However, this latter finding should be interpreted in the light of the fact that no infant had undetectable neutralising antibody to any of the three virus types after three doses of vaccine. The IgG responses to diphtheria and tetanus were similar to that seen with Infanrix-Hib, ${ }^{5}$ with all infants achieving protective titres.

Different vaccine combinations given concurrently have been shown to reduce immune responses to antigens. ${ }^{18}$ In addition, the concurrent administration with Pediacel of an MCC vaccine conjugated to tetanus toxoid in term infants has resulted in an increased PRP IgG GMC but a reduced MCC SBA GMT compared to that seen when an MCC$\mathrm{CRM}_{197}$ vaccine was given. ${ }^{19}$ While a combined DT5aP-HibIPV vaccine appears an attractive option it is clear that further research is needed to determine the optimum combination of aP, Hib, and MCC vaccines in the preterm infant. Future studies will also need to investigate the response to pneumococcal conjugates in this population and the effect such a vaccine might have on responses to other vaccines.

\section{ACKNOWLEDGEMENTS}

We wish to express our gratitude to the parents and infants who have participated in the study, to study nurses Mrs C Schapira, Mrs T Griffen, Mrs J Hovey, and Mrs F McNeight, and to all the consultants in the participating centres for allowing us to investigate their patients.

\section{Authors' affiliations}

M H Slack, S Cade, R J Thwaites, Department of Paediatrics, St Mary's Hospital, Portsmouth, UK

D Schapira, Department of Paediatrics, Royal Hampshire County Hospital, Winchester, UK

A Crowley-Luke, HPA Porton Down, Salisbury, UK 
J Southern, E Miller, Immunisation Division, HPA Communicable Disease Surveillance Centre, 61 Colindale Avenue, London, UK R Borrow, Vaccine Evaluation Department, Manchester Royal Infirmary, Manchester, UK

Funding: The study was funded in part by an unrestricted grant from AventisPasteur MSD

Competing interests: Dr Slack and Ms Southern attended the 2003 Annual Meeting of the European Society of Paediatric Infectious Diseases as guests of AventisPasteur MSD

\section{REFERENCES}

1 Ramsay ME, Miller E, Ashworth LAE, et al. Adverse events and antibody response to accelerated immunisation in term and preterm infants. Arch Dis Child 1995;72:230-2.

2 D'Angio CT, Maniscalco WM, Pichichero ME. Immunologic response of extremely preterm infants to tetanus, Haemophilus influenzae and polio immunizations. Pediatrics 1995;96:18-22.

3 Bernbaum JC, Daft A, Anolik R, et al. Response of preterm infants to diphtheria-tetanus-pertussis immunizations. J Pediatr 1985;107:184-8.

4 Conway S, James J, Balfour A, et al. Immunisation of the preterm baby. J Infect 1993;27:143-50.

5 Slack MH, Schapira D, Thwaites RJ, et al. Acellular pertussis vaccine given by accelerated schedule: preterm infants' response. Arch Dis Child Fetal Neonatal Ed 2004;89:F57-60.

6 Adenyi-Jones SC, Faden H, Ferdon MB, et al. Systemic and local immune responses to enhanced-potency inactivated poliovirus vaccine in premature and term infants. J Pediatr 1992; 120:686-9.

7 Smolen P, Bland R, Heiligenstein E, et al. Antibody response to oral polio vaccine in premature infants. J Pediatr 1983;103:917-19.

8 Linder N, Handsher R, German B, et al. Controlled trial of immune response of preterm infants to recombinant hepatitis $B$ and inactivated poliovirus vaccines administered simultaneously shortly after birth. Arch Dis Child Fetal Neonatal Ed 2000;83:F24-7.

9 D'Angio CT, Maniscalco WM, Pichichero ME. Immunologic response of extremely premature infants to tetanus, Haemophilus influenzae, and polio immunizations. Pediatrics 1995;96:18-20.
10 Slack MH, Schapira D, Thwaites RJ, et al. Immune response of preterm infants to meningococcal serogroup $C$ and combined diphtheria-tetanus toxoidsacellular pertussis-Haemophilus influenzae type $b$ conjugate vaccines. $J$ Infect Dis 2001;184:1617-20.

11 Shinefield H, Black S, Ray P, et al. Efficacy, immunogenicity and safety of heptavalent pneumococcal conjugate vaccine in low birth weight and preterm infants. Pediatr Infect Dis J 2002;21:182-6.

12 Goldblatt D, Richmond P, Millard E, et al. The induction of immunologic memory after vaccination with Haemophilus influenzae type b conjugate and acellular pertussis-containing diphtheria, tetanus, and pertussis combination. $J$ Infect Dis 1999;180:538-41.

13 Eskola J, Olander RM, Hovi T, et al. Randomised trial of the effect of coadministration with acellular pertussis DTP vaccine on immunogenicity of Haemophilus influenzae type b conjugate vaccine. Lancet 1996;348:1688-99.

14 Lee CY, Thipphawong J, Huang LM, et al. An evaluation of the safety and immunogenicity of a five-component acellular pertussis, diphtheria, and tetanus toxoid vaccine (DTaP) when combined with a Haemophilus influenzae type b-tetanus toxoid conjugate vaccine (PRP-T) in Taiwanese infants. Pediatrics 1999; 103:25-30.

15 Richmond P, Borrow R, Miller E, et al. Meningococcal serogroup C conjugate vaccine is immunogenic in infancy and primes for memory. $J$ Infect Dis 1999; 179:1569-72.

16 Olin P, Rasmussen F, Gustafsson L, et al. Randomised controlled trial of two-component, three-component, and five-component acellular pertussis vaccines compared with whole cell pertussis vaccine. Lancet 1997;350:1569-77.

17 McVernon J, Andrews N, Slack MP, et al. Risk of vaccine failure after Haemophilus influenzae type $b$ (Hib) combination vaccines with acellular pertussis. Lancet 2003;361:1521-3.

18 Dagan R, Eskola J, Leclerc C, et al. Reduced response to multiple vaccines sharing common protein epitopes that are administered simultaneously to infants. Infect Immun 1998;66:2093-8.

19 Miller $\mathbf{E}$, Southern J, Kitchin N, et al. Interaction between different meningococcal $\mathrm{C}$ conjugate vaccines and the Hib component of concomitantly administered diphtheria/tetanus/pertussis/Hib vaccines with either whole cell or acellular pertussis antigens. European Society of Paediatric Infectious Diseases, 21 st Annual Meeting, 2003, Abstract 272

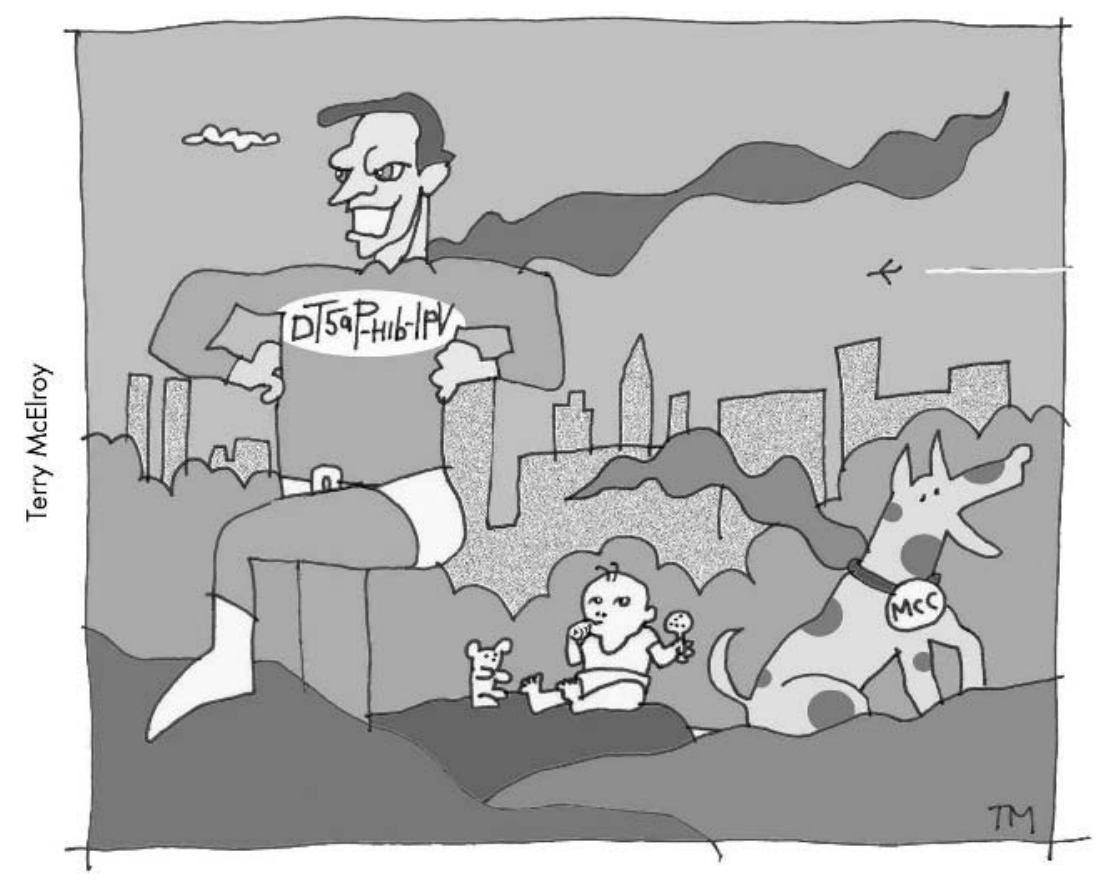

